Graph Theory xx (xxxx) 1-14

\title{
ON THE STRONG PARITY CHROMATIC NUMBER
}

\author{
Július CzAP $^{1}$, Stanislav JENDROL ${ }^{2}$ \\ AND FRANTIŠEK KARDOŠ ${ }^{2}$ \\ ${ }^{1}$ Department of Applied Mathematics and Business Informatics, \\ Faculty of Economics, Technical University of Košice, \\ Němcovej 32, SK-04001 Košice, Slovakia \\ ${ }^{2}$ Institute of Mathematics, P. J. Šafárik University, \\ Jesenná 5, SK-04001 Košice, Slovakia \\ email: julius.czap@tuke.sk \\ \{stanislav.jendrol,frantisek.kardos\}@upjs.sk
}

\begin{abstract}
A vertex colouring of a 2-connected plane graph $G$ is a strong parity vertex colouring if for every face $f$ and each colour $c$, the number of vertices incident with $f$ coloured by $c$ is either zero or odd.

Czap et al. in [9] proved that every 2-connected plane graph has a proper strong parity vertex colouring with at most 118 colours.

In this paper we improve this upper bound for some classes of plane graphs.
\end{abstract}

Keywords: plane graph, $k$-planar graph, vertex colouring, strong parity vertex colouring.

2010 Mathematics Subject Classification: 05C15.

\section{INTRODUCTION}

We adapt the convention that a graph (as a combinatorial object) is $k$-planar if it can be drawn in the plane (on the sphere) so that each its edge is crossed by at most $k$ other edges; such a drawing is then called a $k$-plane graph (a geometrical object). Specially, for $k=0$ we have planar or plane graphs.

If a plane graph $G$ is drawn in the plane $\mathcal{M}$, then the maximal connected regions of $\mathcal{M} \backslash G$ are called the faces of $G$. The facial walk of a face $f$ of 
a connected plane graph $G$ is the shortest closed walk traversing all edges incident with $f$. The size of a face $f$ is the length of its facial walk. Let a $d$-face be a face of size $d$. A 3-face is called a triangle and a face of size at least 4 is called a non-triangle face.

A triangulation is a simple plane graph which contains only 3-faces. A near-triangulation is a simple plane graph which contains at most one non-triangle face.

The degree of a vertex $v$ of a graph $G$ is the number of edges incident with $v$.

Let the set of vertices, edges, and faces of a connected plane graph $G$ be denoted by $V(G), E(G)$, and $F(G)$, respectively, or by $V, E$, and $F$ if $G$ is known from the context.

A $k$-colouring of the graph $G$ is a mapping $\varphi: V(G) \rightarrow\{1, \ldots, k\}$. A colouring of a graph in which no two adjacent vertices have the same colour is a proper colouring. A graph which has a proper $k$-colouring is called $k$-colourable.

Let $\varphi$ be a vertex colouring of a connected plane graph $G$. We say that a face $f$ of $G$ uses a colour $c$ under the colouring $\varphi k$ times if this colour appears $k$ times along the facial walk of $f$. (The first and the last vertex of the facial walk is counted as one appearance only.)

The problems of graph colouring, in particular the well-known Four Colour Problem [14], have motivated the development of different graph colourings, which brought many problems and questions in this area. Colourings of graphs embedded on surfaces with face constraints have recently drawn a substantial amount of attention, see e.g. [4, 5, 10, 11, 12, 16]. Two problems of this kind are the following.

Problem 1. A vertex colouring $\varphi$ is a weak parity vertex colouring of a connected plane graph $G$ if each face of $G$ uses at least one colour an odd number of times. The problem is to determine the minimum number $\chi_{w}(G)$ of colours used in such a colouring of $G$. The number $\chi_{w}(G)$ is called the weak parity chromatic number of $G$.

Problem 2. A vertex colouring $\varphi$ is a strong parity vertex colouring of a 2 -connected plane graph $G$ if for each face $f$ and each colour $c$, either no vertex or an odd number of vertices incident with $f$ is coloured by $c$. The problem is to find the minimum number $\chi_{s}(G)$ of colours used in such a colouring of a given graph $G$. The number $\chi_{s}(G)$ is called the strong parity chromatic number of $G$. 
Our research has been motivated by the paper [6] which deals with parity edge colourings in graphs. Recall that a parity edge colouring is such a colouring in which each walk uses some colour an odd number of times. The parity edge chromatic number $p(G)$ is the minimum number of colours in a parity edge colouring of $G$. Computing $p(G)$ is NP-hard even when $G$ is a tree, but the problem of recognizing parity edge colourings of graphs is solvable in polynomial time. The vertex version of this problem is introduced in [5]. This article deals with parity vertex colourings of plane graphs focused on facial walks.

The first problem has been investigated in [7]. The authors have found a general upper bound for this parameter.

Theorem 1 (Czap and Jendrol [7]). Let $G$ be a 2-connected plane graph. Then there is a proper weak parity vertex 4-colouring of $G$, such that each face of $G$ uses some colour exactly once.

Czap and Jendrol' [7] conjecture that $\chi_{w}(G) \leq 3$ for all simple plane graphs $G$ and they have proved that this conjecture is true for the class of 2-connected simple cubic plane graphs. This conjecture is still open in general.

In this paper, we focus on the second problem.

In the paper [7] there is posed a conjecture that for any 2-connected plane graph $G$ the strong parity chromatic number can be bounded from above by a constant. The conjecture was proved by Czap et al. in the following form.

Theorem 2 (Czap, Jendrol, and Voigt [9]). Let $G$ be a 2-connected plane graph. Then $G$ has a proper strong parity vertex colouring with at most 118 colours.

The constant 118 was recently improved to 97 by Kaiser et al. [13]. In this section, we improve this upper bound for 3-connected simple plane graphs having property that the faces of a certain size are in a sense far from each other.

The following lemma is fundamental. Remind that a cycle can be considered as a connected 2-regular plane graph. 
Lemma 3. Let $C=v_{1}, \ldots, v_{k}$ be a cycle on $k$ vertices. Then there is a proper strong parity vertex colouring $\varphi$ of $C$ using the colours $a, b, c, d, e$, where the colours $a, b, c$ are used at most once.

Proof. We define the colouring $\varphi$ of $C$ in the following way:

- $k=4 t$, then $\varphi\left(v_{1}\right)=a, \varphi\left(v_{2}\right)=b, \varphi\left(v_{i}\right)=d$ for $i \equiv 1(\bmod 2), i>1$, and $\varphi\left(v_{i}\right)=e$ for $i \equiv 0(\bmod 2), i>2$.

- If $k=4 t+1$, then $\varphi\left(v_{1}\right)=a, \varphi\left(v_{2}\right)=b, \varphi\left(v_{3}\right)=c, \varphi\left(v_{i}\right)=d$ for $i \equiv 1$ $(\bmod 2), i>3$, and $\varphi\left(v_{i}\right)=e$ for $i \equiv 0(\bmod 2), i>2$.

- If $k=4 t+2$, then $\varphi\left(v_{i}\right)=d$ for $i \equiv 1(\bmod 2)$ and $\varphi\left(v_{i}\right)=e$ for $i \equiv 0$ $(\bmod 2)$.

- If $k=4 t+3$, then $\varphi\left(v_{1}\right)=a, \varphi\left(v_{i}\right)=d$ for $i \equiv 1(\bmod 2), i>1$, and $\varphi\left(v_{i}\right)=e$ for $i \equiv 0(\bmod 2)$.

Clearly, this colouring satisfies our requirements in each case.

Lemma 4. Let $G$ be a 3-connected near-triangulation. Then there is a proper strong parity vertex colouring of $G$ which uses at most 6 colours. Moreover, this bound is best possible.

Proof. If $G$ is a triangulation, then by the Four Colour Theorem [1] we can colour the vertices of $G$ with at most 4 colours in such a way that the vertices incident with the same face receive different colours. Clearly, this colouring is a strong parity vertex one.

Now we suppose that $G$ contains a $d$-face $f, d \geq 4$. Let $v_{1}, \ldots, v_{d}$ be the vertices incident with $f$ in this order. Next we insert the diagonals $v_{1} v_{i}$, $i \in\{3, \ldots, d-1\}$ and we get a new graph $T$. The graph $T$ has a proper colouring which uses at most four colours, since it is a plane triangulation. This colouring induces the colouring $\varphi$ of $G$ in the natural way.

We can assume that $\varphi\left(v_{1}\right)=1, \varphi\left(v_{2}\right)=2$, and $\varphi\left(v_{3}\right)=3$. Next we use Lemma 3 and we recolour the vertices incident with the face $f$. We use the following colours: $a=1, b=2, c=3, d=5$, and $e=6$.

Observe, that each triangle face of $G$ uses three different colours and from Lemma 3 it follows that the face $f$ uses each colour which appears on its boundary an odd number of times.

To see that the bound 6 is best possible it suffices to consider the graph of a wheel $W_{5}$ depicted in Figure 1.

We write $v \in f$ if a vertex $v$ is incident with a face $f$. Two distinct faces $f$ and $g$ touch each other, if there is a vertex $v$ such that $v \in f$ and $v \in g$. 


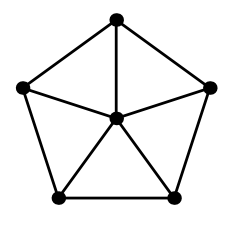

Figure 1: An example of a graph with no proper strong parity vertex colouring using less than 6 colours.

Two distinct faces $f$ and $g$ influence each other, if they touch, or there is a face $h$ such that $h$ touches both $f$ and $g$.

Theorem 5. Let $G$ be a 3-connected plane graph in which no two nontriangle faces influence each other. Then there is a proper strong parity vertex colouring of $G$ which uses at most six colours $1, \ldots, 6$ such that each vertex which is not incident with any non-triangle face has a colour from the set $\{1,2,3,4\}$. Moreover, this bound is sharp.

Proof. We apply induction on the number of non-triangle faces. If $G$ contains one non-triangle face then the claim follows from Lemma 4.

Assume that $G$ contains $j$ non-triangle faces, $j \geq 2$. Let $f=v_{1}, \ldots, v_{m}$ be one of them. We insert the diagonals $v_{1} v_{i}, i \in\{3, \ldots, m-1\}$ to the face $f$ and we get a new graph $H$. The graph $H$ has $(j-1)$ non-triangle faces, hence, by induction, it has a proper strong parity vertex colouring which uses at most six colours $1, \ldots, 6$. Moreover, each vertex which is not incident with any non-triangle face has a colour from the set $\{1,2,3,4\}$. This colouring of $H$ induces the colouring $\varphi$ of $G$.

Observe, that the vertices incident with $f$ or the faces which touch $f$ have colours from the set $\{1,2,3,4\}$ (else $G$ contains two non-triangle faces that influence each other). We use the colouring from Lemma 3 with the colours $a=\varphi\left(v_{1}\right), b=\varphi\left(v_{2}\right), c=\varphi\left(v_{3}\right), d=5$, and $e=6$ to recolour the vertices incident with $f$ so that we obtain a required colouring of $G$.

To see that the bound 6 is best possible it suffices to consider a triangulation $T$ such that it contains $\ell$ triangle faces $f_{1}, f_{2}, \ldots, f_{\ell}$ which do not influence each other, and insert a wheel-like configuration into each of them, see Figure 2 for illustration. 

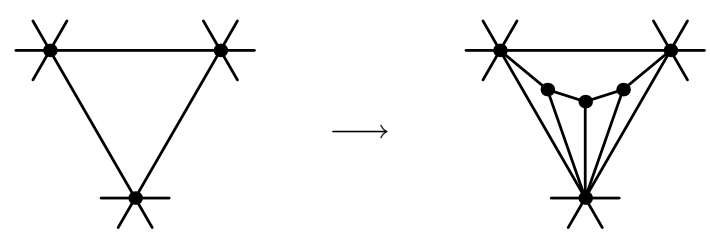

Figure 2: Inserting a path on three vertices into a triangle face yields a configuration without a required colouring using less than 6 colours.

\subsection{Strong PARIty COLOURING Versus CyCliC COLOURING}

A cyclic colouring of a plane graph is a vertex colouring in which, for each face $f$, all the vertices on the boundary of $f$ have distinct colours. The cyclic chromatic number $\chi_{c}(G)$ of a plane graph $G$ is the minimum number of colours in a cyclic colouring. Clearly, every cyclic colouring of a 2-connected plane graph is also a strong parity vertex colouring, hence, $\chi_{s}(G) \leq \chi_{c}(G)$. Therefore, every upper bound on $\chi_{c}(G)$ also applies for $\chi_{s}(G)$. There are several known bounds on $\chi_{c}(G)$ depending on $\Delta^{*}(G)$, the maximum face size of a plane graph $G$. The results of [16], [11], [12], and [10] immediately give the following statements.

Proposition 6. Let $G$ be a 2-connected plane graph with maximum face size $\Delta^{*}$. Then

$$
\chi_{s}(G) \leq\left\lceil\frac{5 \Delta^{*}}{3}\right\rceil .
$$

Moreover, if $G$ is 3-connected, then

$$
\chi_{s}(G) \leq\left\{\begin{array}{lll}
\Delta^{*}+1 & \text { for } & \Delta^{*} \geq 60 \\
\Delta^{*}+2 & \text { for } & \Delta^{*} \geq 18 \\
\Delta^{*}+5 & \text { for } & \Delta^{*} \geq 3
\end{array}\right.
$$

Borodin et al. proved the following.

Theorem 7 (Borodin [2]). Let $G$ be a 2-connected plane graph with maximum face size $\Delta^{*} \leq 4$. Then $\chi_{c}(G) \leq 6$.

Theorem 8 (Borodin, Sanders and Zhao [4]). Let $G$ be a 2-connected plane graph with maximum face size $\Delta^{*} \leq 5$. Then $\chi_{c}(G) \leq 8$.

We use these theorems to improve the general upper bound on $\chi_{s}(G)$ for several graph classes with arbitrary large faces. 
Theorem 9. Let $G$ be a 3-connected plane graph in which the faces of size at least 5 do not influence each other. Then there is a proper strong parity vertex colouring of $G$ which uses at most 8 colours.

Proof. Let $B=\left\{f_{1}, \ldots, f_{\ell}\right\}$ be the set of faces of size at least 5 and let $d_{i}$ denote the size of the face $f_{i}$. Let the face $f_{i}$ be incident with the vertices $v_{i, 1}, \ldots, v_{i, d_{i}}, i \in\{1, \ldots, \ell\}$. Next we insert the diagonals $v_{i, 1} v_{i, m}, m \in$ $\left\{3, \ldots, d_{i}-1\right\}$, to the face $f_{i}$ for $i \in\{1, \ldots, \ell\}$, and we get a new graph $H$. Observe, that $H$ contains only 3 -faces and 4 -faces.

¿From Theorem 7 it follows that $H$ has a cyclic colouring with at most six colours. This colouring defines the colouring $\varphi$ of $G$. Clearly, each face of $G$ of size $j, j \in\{3,4\}$, uses $j$ different colours. Finally, we recolour the vertices incident with the faces from $B$ in such a way that we get a proper strong parity vertex colouring of $G$. For the face $f_{i}$ we use the same colouring as in Lemma 3 with $a=\varphi\left(v_{i, 1}\right), b=\varphi\left(v_{i, 2}\right), c=\varphi\left(v_{i, 3}\right), d=7, e=8$.

It is easy to check that this colouring of $G$ satisfies our requirements.

Theorem 10. Let $G$ be a 3-connected plane graph such that the faces of size at least 6 do not influence each other. Then there is a proper strong parity vertex colouring of $G$ which uses at most 10 colours.

Proof. We create a graph $H$ from $G$ analogously as in the proof of Theorem 9. Using Theorem 8 we colour the vertices of $H$ cyclically with at most 8 colours. By this colouring we get the colouring $\varphi$ of $G$.

At this time each face of $G$ of size $j, j \in\{3,4,5\}$, uses $j$ different colours. We recolour the vertices incident with $f_{i}$ by the colouring defined in Lemma 3 . We use the following colours: $a=\varphi\left(v_{i, 1}\right), b=\varphi\left(v_{i, 2}\right), c=\varphi\left(v_{i, 3}\right), d=9$, $e=10$.

Recall that a graph is $k$-planar if it can be drawn in the plane so that each its edge is crossed by at most $k$ other edges. In this section we investigate the structure of $k$-planar graphs. We will use only one operation, namely the contraction. The contraction of an edge $e=u v$ in the graph $G$, denoted by $G \circ e$, is defined as follows: identify the vertices $u$ and $v$, delete the loop $u v$ and replace all multiple edges arisen by single edges. 
Lemma 11. Let $G$ be a drawing of a k-planar graph, and let e be an edge which is not crossed by any other edge. Then $G \circ e$ is a k-planar graph.

Proof. While contracting the edge $e$, the number of crossings of any edge does not increase, therefore, the graph remains $k$-planar.

Lemma 12. Let $G$ be a drawing of a $k$-planar graph, and let $C=v_{1}, \ldots, v_{t}$ be a cycle in $G$ such that the edges $v_{i} v_{i+1}, i \in\{1, \ldots, t\}, v_{t+1}=v_{1}$, are not crossed by any other edge and the inner part of $C$ does not contain any vertex. Let $H$ be a graph obtained from $G$ by collapsing $C$ into a single vertex (and replacing multiple edges by single edges). Then the graph $H$ is a k-planar graph.

Proof. We successively contract the edges $v_{1} v_{2}, \ldots, v_{t-1} v_{t}$. After the contraction of $v_{1} v_{2}$ we obtain a $k$-planar graph (see Lemma 11). Clearly, there exists a plane drawing of $G$ such that the edges on the cycle corresponding to $C$ are not crossed by any other edge and the cycle has an empty inner part. When we contract the last edge $v_{t-1} v_{t}$ we get the graph $H$.

We say that a face $f$ of size $i$ is isolated if there is no face $g$ of size at least $i$ touching $f$.

Lemma 13. Let $j$ be a fixed integer from the set $\{3,4,5\}$. Let $G$ be a 2connected plane graph such that any face of size at least $j+1$ is isolated. Let $H$ be a graph obtained from $G$ in the following way: for each face in $G$ of size at least $j+1$ insert a vertex to $H$, join two vertices of $H$ by an edge if the corresponding faces influence each other in $G$. Then

1 If $j=3$ then $H$ is a planar graph.

2 If $j=4$ then $H$ is a 1-planar graph.

3 If $j=5$ then $H$ is a 2-planar graph.

Proof. Let $B=\left\{f_{1}, \ldots, f_{\ell}\right\}$ be a set of faces which have sizes at least $j+1$. Let $V\left(f_{i}\right)$ denote the set of vertices of $G$ incident with the face $f_{i}$, $i \in\{1, \ldots, \ell\}$. Clearly, $V\left(f_{i}\right) \cap V\left(f_{j}\right)=\emptyset$, for $i \neq j$, because $f_{i}$ and $f_{j}$ do not touch each other.

Given the sets $V\left(f_{i}\right)$, we colour the vertices of $G$ in the following way: Vertices contained in $V\left(f_{i}\right)$ receive the colour $i$; vertices not contained in any $V\left(f_{i}\right)$ receive the colour 0 .

To each face $g$ with a facial walk $u_{1}, \ldots, u_{p}, 4 \leq p \leq j$ we insert the diagonal $u_{n} u_{m}, n, m \in\{1, \ldots, p\}$, if the vertices $u_{n}$ and $u_{m}$ have distinct 
colours and these colours are different from 0 . So we get the graph $G_{1}$. Let $G_{2}$ be a graph induced on the vertices of $G_{1}$ which have colours different from 0 and let $G_{3}$ be a graph obtained from $G_{2}$ by collapsing the vertices from $V\left(f_{i}\right)$ to the vertex $v_{i}, i \in\{1, \ldots, \ell\}$.

Observe that,

1. If $j=3$ then $G=G_{1}$, hence $G_{2}$ is a plane graph. From Lemma 12 it follows that $G_{3}$ is a plane graph.

2. If $j=4$ then to each face of size 4 we add at most 2 diagonals, hence, $G_{1}$ is a 1-plane graph. $G_{2}$ is a subgraph of $G_{1}$ therefore it is 1-plane too. Lemma 12 ensures that $G_{3}$ is 1-plane.

3. If $j=5$ then $G_{1}$ and $G_{2}$ are 2-plane graphs because the complete graph on 5 vertices is 2-planar. From Lemma 12 it follows that $G_{3}$ is 2-plane.

Observe, the vertices $v_{s}, v_{t}$ of $G_{3}, s, t \in\{1, \ldots, \ell\}$, are joined by an edge if and only if the corresponding faces $f_{s}, f_{t}$ of $G$ influence each other. Hence, the graph $G_{3}$ is the plane drawing of $H$.

In the rest of the paper let $B_{i}(G)$ (or $B_{i}$ if $G$ is known from the context) denote the set of faces of $G$ of size at least $i, i \in\{4,5,6\}$, and let $\ell_{i}$ denote the number of faces in $B_{i}(G)$. Let $H_{i}$ be a graph obtained from $G$ in the following way: for each face $f \in B_{i} \subseteq F(G)$ insert a vertex to $H_{i}$, join two vertices of $H_{i}$ if the corresponding faces influence each other in $G$.

The previous theorems give upper bounds for the strong parity chromatic number for graphs in which any two faces of size at least 4, 5 or 6 do not influence each other. In the next part of this article we provide another upper bound in the case when the faces of size at least six do not touch but they can influence one another.

Theorem 14. Let $G$ be a 3-connected plane graph such that any face of size at least 4 is isolated. Then there is a proper strong parity vertex colouring of $G$ which uses at most 12 colours.

Proof. If $G$ does not contain any two non-triangle faces influencing each other then from Theorem 5 it follows that $G$ has a required colouring.

Assume that $G$ contains at least two non-triangle faces which influence each other. Let the face $f_{i} \in B_{4}$ be incident with the vertices $v_{i, 1}, \ldots, v_{i, d_{i}}$, $i \in\left\{1, \ldots, \ell_{4}\right\}$, where $d_{i}$ is the size of $f_{i}$. We insert the diagonals $v_{i, 1} v_{i, m}$, $m \in\left\{3, \ldots, d_{i}-1\right\}$, to the face $f_{i}$ for $i \in\left\{1, \ldots, \ell_{4}\right\}$, and we get a triangulation $T$. Using the Four Colour Theorem we colour the vertices of $T$ with at most four colours such that adjacent vertices receive distinct colours. This colouring induces the colouring $\varphi$ of $G$. 
¿From Lemma 13 it follows that $H_{4}$ is a planar graph, hence, we can assign to each vertex of $H_{4}$ one pair of colours from $\{(5,6),(7,8),(9,10),(11,12)\}$ in such a way that two adjacent vertices receive distinct pairs. It means that we can assign a pair of colours to each face of $G$ of size at least four in such a way that two faces which influence each other receive distinct pairs.

Assume that we assigned the pair $\left(x_{i}, y_{i}\right)$ to the face $f_{i}$. Now we recolour the vertices incident with $f_{i}, i \in\left\{1, \ldots, \ell_{4}\right\}$. We use the same colouring as in Lemma 3 with colours $a=\varphi\left(v_{i, 1}\right), b=\varphi\left(v_{i, 2}\right), c=\varphi\left(v_{i, 3}\right), d=x_{i}$, and $e=y_{i}$. If we perform this recolouring of vertices on all faces of size at least 4 we obtain such a colouring that if a colour appears on a face $f_{i} \in B_{4}$, $i \in\left\{1, \ldots, \ell_{4}\right\}$, then it appears an odd number of times. Moreover, if we recolour at least two vertices on a triangle face of $G$ then we recolour them with distinct colours, because the corresponding faces influence each other.

There is a lot of papers about plane graphs and their colourings but little is known about $k$-planar graphs, $k \geq 1$. We use the following result of Borodin to find an upper bound on $\chi_{s}(G)$ for the class of 3-connected plane graphs for which the faces of size at least five are in a sense far from each other.

Theorem 15 (Borodin [3]). If a graph is 1-planar, then it is vertex 6colourable.

Theorem 16. Let $G$ be a 3-connected plane graph such that any face of size at least 5 is isolated. Then there is a proper strong parity vertex colouring of $G$ which uses at most 18 colours.

Proof. Assume that $G$ contains at least two faces of size at least 5 which influence each other. Let the face $f_{i} \in B_{5}$ be incident with the vertices $v_{i, 1}, \ldots, v_{i, d_{i}}, i \in\left\{1, \ldots, \ell_{5}\right\}$, where $d_{i}$ is the size of $f_{i}$. Next we insert the diagonals $v_{i, 1} v_{i, m}, m \in\left\{3, \ldots, d_{i}-1\right\}$, to the face $f_{i}$ for $i \in\left\{1, \ldots, \ell_{5}\right\}$, and we get a graph $W$. Observe, that each face of $W$ has size at most 4 . Applying Theorem 7 we colour the vertices of $W$ with at most 6 colours cyclically. This colouring defines the colouring $\varphi$ of $G$.

From Lemma 13 it follows that $H_{5}$ is a 1-planar graph. By Theorem 15 we can assign to each vertex of $H_{5}$ one pair of colours from $\{(7,8), \ldots,(17,18)\}$ so that two adjacent vertices receive distinct pairs. Ergo, we assign distinct pairs of colours to faces of $G$ of size at least 5 which influence each other.

Assume that the face $f_{i}$ receives the pair $\left(x_{i}, y_{i}\right)$. Now we recolour the vertices incident with $f_{i}$ by the colouring defined in Lemma 3 . We use the following colours: $a=\varphi\left(v_{i, 1}\right), b=\varphi\left(v_{i, 2}\right), c=\varphi\left(v_{i, 3}\right), d=x_{i}$, and $e=y_{i}$. 
If we perform this recolouring of vertices on all faces of size at least 5 we obtain a required colouring of $G$.

The class of 2-planar graphs has not been sufficiently investigated. Pach and Tóth tried to answer the following question: What is the maximum number of edges that a simple graph of $n$ vertices can have if it can be drawn in the plane so that every edge crosses at most $k$ others? They proved the following.

Theorem 17 (Pach and Tóth [15]). Let $G$ be a simple graph drawn in the plane so that every edge is crossed by at most $k$ others. If $0 \leq k \leq 4$, then we have $|E(G)| \leq(k+3) \cdot(|V(G)|-2)$.

Using this result we can prove that every 2-planar graph has a vertex of degree at most 9 , therefore 2-planar graphs are 10-colourable. In the next lemma let $\delta(G)$ denote the minimum vertex degree of a graph $G$.

Lemma 18. Let $G$ be a 2-planar graph. Then $\delta(G) \leq 9$.

Proof. ¿From Theorem 17 it follows that $|E(G)| \leq 5 \cdot|V(G)|-10$. For every graph it holds $2 \cdot|E(G)|=\sum_{v \in V(G)} \operatorname{deg}(v) \geq \delta(G) \cdot|V(G)|$. Hence, we get

$$
\begin{gathered}
10 \cdot|V(G)|-20 \geq|V(G)| \cdot \delta(G) \\
\delta(G) \leq \frac{10 \cdot|V(G)|-20}{|V(G)|}<10 .
\end{gathered}
$$

Corollary 19. If a graph is 2-planar, then it is vertex 10-colourable.

This information about 2-planar graphs helps us to prove the following theorem.

Theorem 20. Let $G$ be a 3-connected plane graph such that any face of size at least 6 is isolated. Then there is a proper strong parity vertex colouring of $G$ which uses at most 28 colours.

Proof. The proof follows the scheme of the proof of Theorem 16. We omit the details. 


\section{Applichtions}

Two edges of a plane graph are face-adjacent if they are consecutive edges of a facial walk of some face. The facial parity edge colouring of a connected bridgeless plane graph is an edge colouring such that no two face-adjacent edges receive the same colour, and for each face $f$ and each colour $c$, either no edge or an odd number of edges incident with $f$ is coloured by $c$. The minimum number of colours $\chi_{f p}^{\prime}(G)$ used in such a colouring is called the facial parity chromatic index of $G$. In [8] it is proved that $\chi_{f p}^{\prime}(G) \leq 92$ for an arbitrary connected bridgeless plane graph $G$.

The medial graph $M(G)$ of a plane graph $G$ is obtained as follows. For each edge $e$ of $G$ insert a vertex $m(e)$ in $M(G)$. Join two vertices of $M(G)$ if the corresponding edges are face-adjacent (see [14], pp. 47).

Lemma 21. Let $G$ be a 3-connected plane graph. Then the graph $M(G)$ is 3-connected too.

Proof. By contradiction, suppose that $m\left(e_{1}\right)$ and $m\left(e_{2}\right)$ form a 2-vertexcut in $M(G)$. Let $M_{1}, M_{2}$ be the components of $M(G) \backslash\left\{m\left(e_{1}\right), m\left(e_{2}\right)\right\}$; let $E_{1}$ and $E_{2}$ be the corresponding decomposition of $E(G) \backslash\left\{e_{1}, e_{2}\right\}$. Let the edges from $E_{1}$ (resp. $E_{2}$ ) be white (resp. black); let $e_{1}$ and $e_{2}$ be red.

Let $V_{i}$ be the set of vertices incident only with edges from $E_{i} \cup\left\{e_{1}, e_{2}\right\}$, $i=1,2$. Since the minimum degree of $G$ is at least 3 , we have $V_{1} \cap V_{2}=\emptyset$. If $V_{1} \cup V_{2}=V(G)$, then there are no vertices incident both with white and black edges. Hence, $\left\{e_{1}, e_{2}\right\}$ is a 2-edge-cut in $G$, which is not possible since $G$ is 3-connected.

Therefore, $V_{1} \cup V_{2} \neq V(G)$; let $v$ be a vertex incident both with a white and a black edge. Since $m\left(e_{1}\right), m\left(e_{2}\right)$ is a 2 -vertex-cut in $M(G)$ no white edge is face-adjacent to any black edge in $G$. Hence $v$ has to be incident to both red edges $e_{1}$ and $e_{2}$. Then $V(G) \backslash\left(V_{1} \cup V_{2}\right)=\{v\}$, unless $e_{1}$ and $e_{2}$ are parallel edges in $G$. Let $e_{1}=u v$. We may assume $u \in V_{1}$, i.e. all edges incident with $u$ but $e_{1}$ are white. Then on the boundary cycle of (at least) one of the faces incident with $e_{1}$ white and black edges meet, which is a contradiction.

Observe that every proper strong parity vertex colouring of $M(G)$ corresponds to the facial parity edge colouring of a 3-connected plane graph $G$. We can immediately derive the following upper bounds for the facial parity chromatic index for some classes of plane graphs from Theorems 5, 9, 10, 14,16 , and 20 . 
Corollary 22. (a) Let $G$ be a 3-connected plane graph such that the nontriangle faces of $M(G)$ do not influence each other. Then $\chi_{f p}^{\prime}(G) \leq 6$.

(b) Let $G$ be a 3-connected plane graph such that the faces of $M(G)$ of size at least 5 do not influence each other. Then $\chi_{f p}^{\prime}(G) \leq 8$.

(c) Let $G$ be a 3-connected plane graph such that the faces of $M(G)$ of size at least 6 do not influence each other. Then $\chi_{f p}^{\prime}(G) \leq 10$.

(d) Let $G$ be a 3-connected plane graph such that any face of $M(G)$ of size at least 4 is isolated. Then $\chi_{f p}^{\prime}(G) \leq 12$.

(e) Let $G$ be a 3-connected plane graph such that any face of $M(G)$ of size at least 5 is isolated. Then $\chi_{f p}^{\prime}(G) \leq 18$.

(f) Let $G$ be a 3-connected plane graph such that any face of $M(G)$ of size at least 6 is isolated. Then $\chi_{f p}^{\prime}(G) \leq 28$.

\section{Acknowledgment}

This work was supported by the Slovak Science and Technology Assistance Agency under the contract No APVV-0007-07.

\section{REFERENCES}

[1] K. Appel and W. Haken, Every planar map is four colorable, Bull. Amer. Math. Soc. 82 (1976) 711-712.

[2] O.V. Borodin, Solution of Ringel's problems on vertex-free coloring of plane graphs and coloring of 1-planar graphs, , Met. Diskret. Anal. 41 (1984) 12-26 (in Russian).

[3] O.V. Borodin, A new proof of the 6 color theorem, J. Graph Theory 19 (1995) $507-521$.

[4] O.V. Borodin, D.P. Sanders, and Y. Zhao, On cyclic coloring and their generalizations, Discrete Math. 203 (1999) 23-40.

[5] P. Borowiecki, K. Budajová, S. Jendrol', and S. Krajči, Parity vertex colouring of graphs, Discuss. Math. Graph Theory 31 (2011) 183-195.

[6] D.P. Bunde, K. Milans, D.B. West, and H. Wu, Parity and strong parity edgecoloring of graphs, Congressus Numerantium 187 (2007) 193-213.

[7] J. Czap and S. Jendrol', Colouring vertices of plane graphs under restrictions given by faces, Discuss. Math. Graph Theory 29 (2009) 521-543.

[8] J. Czap, S. Jendrol', and F. Kardoš, Facial parity edge colouring, Ars Math. Contemporanea 4 (2011) 255-269. 
[9] J. Czap, S. Jendrol', and M. Voigt, Parity vertex colouring of plane graphs, Discrete Math. 311 (2011) 512-520.

[10] H. Enomoto and M. Horňák, A general upper bound for the cyclic chromatic number of 3-connected plane graphs, J. Graph Theory 62 (2009) 1-25.

[11] H. Enomoto, M. Horňák, and S. Jendrol, Cyclic chromatic number of 3connected plane graphs, SIAM J. Discrete Math. 14 (2001) 121-137.

[12] M. Horňák and J. Zlámalová, Another step towards proving a conjecture by Plummer and Toft, Discrete Math. 310 (2010) 442-452.

[13] T. Kaiser, O. Rucký, M. Stehlík, and R. Škrekovski, Strong parity vertex coloring of plane graphs, IMFM, Preprint series 49 (2011), 1144.

[14] O. Ore, The Four-color Problem (Academic Press, New York, 1967).

[15] J. Pach and G. Tóth, Graphs drawn with few crossings per edge, Combinatorica 17 (1997) 427-439.

[16] D.P. Sanders and Y. Zhao, A new bound on the cyclic chromatic number, J. Combin. Theory (B) 83 (2001) 102-111.

Received 14 September 2009 Revised 23 May 2011 Accepted 24 May 2011 